\title{
Symmetric States in Quantum Geometry
}

\author{
M. Bojowald and H. A. Kastrup \\ Institute for Theoretical Physics, RWTH Aachen \\ D-52056 Aachen, Germany
}

\begin{abstract}
Symmetric states are defined in the kinematical sector of loop quantum gravity and applied to spherical symmetry and homogeneity. Consequences for the physics of black holes and cosmology are discussed.
\end{abstract}

\section{Introduction}

In the kinematical sector of loop quantum gravity, states are represented as functions on the space $\overline{\mathcal{A}}$ of generalized connections on a principal fiber bundle $P(\Sigma, G)$. Here, $G=S U(2)$ is the gauge group (which can be replaced with any other compact Lie group in the following) and $\Sigma$ the space manifold. All these states can be decomposed in terms of the spin network basis which are special states associated with graphs in $\Sigma$. Given a symmetry group $S$ acting on $\Sigma$, we can ask for states which are symmetric with respect to that action and therefore can be used to study the full theory in a simpler regime. However, as the decomposition into spin networks shows, no ordinary non-trivial state can be exactly symmetric: the discrete structure of space breaks any continuous symmetry. Nevertheless, we can look for symmetric generalized states which is automatic in our definition [1]:

Definition. A symmetric state is a distribution on $\overline{\mathcal{A}}$ whose support contains only connections being invariant under the action of the symmetry group.

In order to describe symmetric states more explicitly, we need more information about invariant connections. A well-known example is that of an $S U(2)$-connection which is invariant under the rotation group:

$\begin{aligned} A_{a}^{i}(x, \vartheta, \varphi) \mathrm{d} x^{a} \tau_{i}= & a(x) \mathrm{d} x \tau_{3}+\cos \vartheta \mathrm{d} \varphi \tau_{3}+\left(-\phi_{1}(x) \mathrm{d} \vartheta+\phi_{2}(x) \sin \vartheta \mathrm{d} \varphi\right) \tau_{1} \\ & -\left(\phi_{2}(x) \mathrm{d} \vartheta+\phi_{1}(x) \sin \vartheta \mathrm{d} \varphi\right) \tau_{2} .\end{aligned}$

*e-mail address: bojowald@physik.rwth-aachen.de

$\dagger$ e-mail address: kastrup@physik.rwth-aachen.de 
It can be decomposed into a reduced connection given by $a$ and scalar fields $\phi_{1 / 2}$ which are functions on the radial reduced manifold $B:=\Sigma / S$. This is in fact the general situation [2, 3]: invariant connections can be classified by a reduced connections and scalar fields on a reduced bundle $Q$ over the reduced manifold $B:=\Sigma / S$. However, in general the structure group of $Q$ is only a subgroup of $G$ (as in the above example because the ansatz (1) is not gauge invariant) which leads to a partial gauge fixing in the classical description. We do not need to care about this partial gauge fixing here because it can be undone in the quantum theory [1] where generalized connections and scalar fields are used which do not rely on using a fixed fiber bundle.

So we arrive at a convenient representation of symmetric states as functions on the space of generalized connections and scalar fields on the reduced manifold. A basis for these states is given by spin network states with Higgs field vertices in the reduced manifold with gauge group $G$. The interpretation of these states is twofold: First, we can use them in order to restrict the full theory to reduced models given by a symmetry condition. This results in a loop quantization of a mini- or midi-superspace model but has the advantage over usual mini-/midi-superspace quantizations that loop quantization methods can be directly applied resulting in a better comparison between a model and the full theory. Second, due to their very definition symmetric states can be seen as distributional states in the full theory. Although they are in this sense idealized, they can be approximated (in the weak topology) by ordinary states which have the interpretation of weave states describing small

perturbations (which are necessary due to quantum fluctuations) around a symmetric (e.g. homogeneous) geometry.

\section{Spherical Symmetry}

Spherically symmetric states are described by spin networks with Higgs field vertices in a one-dimensional (radial) manifold $B$ (see Fig. 1). The Higgs field vertices can be interpreted as representing edges being transversal to orbits of the symmetry group. In the radial manifold a spherical surface is represented by a single point in $B$, the radius $x$, and so it intersects a given spin network state in only one point.

Denoting the radial dreibein components as $E_{i}$, the area of a surface with 


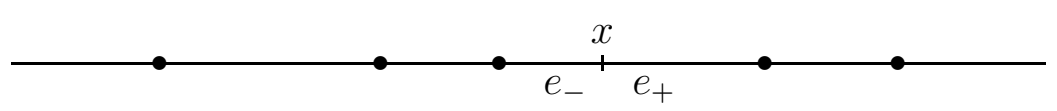

Figure 1: A spherically symmetric spin network.

radius $x$ is classically given by

$$
A(x)=4 \pi \sqrt{\delta^{i j} E_{i}(x) E_{j}(x)} .
$$

It can be quantized along the lines of the full theory [4 by turning the dreibein components into functional derivatives with respect to the radial connection components $A^{i}(x)$. Acting on a spin network function $T$ only the edges $e_{+}$and $e_{-}$(which are assumed to be outgoing in $x$ ) contribute via the holonomies $h_{e_{ \pm}}=\mathcal{P} \exp \int_{e_{ \pm}} \mathrm{d} x A^{i} \tau_{i}$. Using the chain rule we obtain ( $\gamma$ is the Immirzi parameter and $l_{\mathrm{P}}$ the Planck length)

$$
\begin{aligned}
\hat{E}_{i}(x) T & =\frac{\gamma l_{\mathrm{P}}^{2}}{4 \pi i} \sum_{\epsilon \in\{+,-\}} \int_{e_{\epsilon}} \mathrm{d} y \delta(x, y)\left(\tau_{i} h_{e_{\epsilon}}\right)_{B}^{A} \frac{\partial}{\partial\left(h_{e_{\epsilon}}\right)_{B}^{A}} T \\
& =\frac{\gamma l_{\mathrm{P}}^{2}}{4 \pi} \sum_{\epsilon \in\{+,-\}} \frac{\epsilon}{2} J_{e_{\epsilon}}^{i} T
\end{aligned}
$$

where $J_{e}=-i X_{e}$ is given by the right invariant vector field on the copy of $S U(2)$ associated with the edge $e$.

Inserted into the area $A(x)$ this yields

$$
\hat{A}(x)=\frac{1}{2} \gamma l_{\mathrm{P}}^{2} \sqrt{\left(J_{e_{+}}-J_{e_{-}}\right)^{2}}=\frac{1}{2} \gamma l_{\mathrm{P}}^{2} \sqrt{2 J_{e_{+}}^{2}+2 J_{e_{-}}^{2}-\left(J_{e_{+}}+J_{e_{-}}\right)^{2}}
$$

which can be simplified if $x$ is not a Higgs vertex because then gauge invariance implies $J_{e_{+}}+J_{e_{-}}=0$. As compared to the area operator in the full theory, the only difference is that the sum over punctures disappears because in the spherically symmetric sector there is only one puncture. Thus, we have the area spectrum (ignoring Higgs vertices)

$$
A_{j}=\gamma l_{\mathrm{P}}^{2} \sqrt{j(j+1)} \quad, \quad j \in \frac{1}{2} N_{0} .
$$

It is immediate to see that for large values of $j$ it is equidistant and compatible with the Bekenstein spectrum [5] for the horizon area of spherically 
symmetric black holes. On the contrary, the full area spectrum in loop quantum gravity has an exponentially decreasing level distance [四. We can interpret the large difference of the two spectra in the spherically symmetric and the non-symmetric regime as a level splitting familiar from the spectroscopy of atoms: breaking the spherical symmetry leads to a splitting of the levels resulting in an almost dense spectrum (see Fig. 2). A necessary requirement for this to happen is a huge degeneracy of the levels in the spherically symmetric sector which is also expected from thermodynamical considerations: in order to lead to a black hole entropy proportional to the horizon area, the degeneracy of levels in the Bekenstein spectrum has to grow exponentially [6]. However, in quantum geometry spherically symmetric states are distributional and so their degeneracy is not well defined which prohibits a simple counting of states.

\section{Loop Quantum Cosmology}

In specializing the framework to homogeneous or even isotropic states we have the basics of loop quantum cosmology [7]. In this case, the reduced manifold is a single point and so homogeneous states are defined only in terms of scalar fields (point holonomies [8]). For Bianchi models (anisotropic) we have three independent point holonomies which can be visualized as being associated with three closed edges meeting in a single 6-vertex. For isotropic models there is only one point holonomy, but here the concept of spin networks has to be generalized in order to describe all states: the configuration space is no longer a group due to a condition on the scalar fields in an invariant connection if the symmetry group has a non-trivial isotropy subgroup. Therefore, the Peter-Weyl theorem which tells us that functions on a compact group can be expanded into functions associated with matrix elements of all its irreducible representations (which are just spin networks if the group is a product of some copies of $S U(2)$ ) does not apply and a substitute for the expansion has to be found by different means. In the simple case of gauge invariant isotropic states, which depend on only one parameter, it turns out that one can describe all those states as spin networks associated with a single closed edge but possibly with an insertion in the vertex [9]. In this way, the number of states is doubled as compared to a naive expectation. 


\subsection{Volume}

Again, we can observe the phenomenon of level splitting, this time for the volume operator [9]. For Bianchi models, the situation is similar to the area operator in the spherically symmetric sector: the sum over vertices disappears because there is only one 6-vertex to which the point holonomies are attached. For isotropic states, the operator simplifies so much that the complete volume spectrum can be computed explicitly ( $V_{0}$ is an arbitrary constant entering via a homogeneous auxiliary metric):

$$
V_{j}=\gamma^{\frac{3}{2}} V_{0}^{-\frac{1}{2}} l_{\mathrm{P}}^{3} \sqrt{j\left(j+\frac{1}{2}\right)(j+1)} \quad, \quad j \in \frac{1}{2} N_{0} .
$$

The fact that homogeneous states are distributional also implies that there is a discrepancy between mini-superspace quantizations and approximations (by weaves) in the full theory: even small inhomogeneous perturbations cause a transition to the full volume spectrum. This is in contrast to the treatment of inhomogeneities in more standard quantum cosmological models [10] where symmetric and slightly perturbed geometries are smoothly connected.

\subsection{Dynamics}

In cosmological models there is a familiar procedure to study intrinsic dynamics by introducing the volume as internal time. The volume quantization in quantum geometry suggests that such a procedure in loop quantum cosmology leads to a discrete time. This can in fact be made more precise by implementing the intrinsic dynamics into a loop quantization of cosmological models. To that end we need, in the first place, a quantization of the Hamiltonian constraint for cosmological models [11] which can be derived using the key steps of the quantization in the full theory [12 with suitable adaptations in order to respect the symmetry.

The result for Bianchi models is a constraint operator which looks very similar to that in the full theory: the Euclidean part is

$$
\hat{\mathcal{H}}^{(\mathrm{E})}[N]=-4 i\left(\gamma l_{\mathrm{P}}^{2}\right)^{-1} V_{0} N \sum_{I J K} \epsilon^{I J K} \operatorname{tr}\left(h_{I} h_{J} h_{I}^{-1} h_{J}^{-1} h_{[I, J]}^{-1}\left[h_{K}, \hat{V}\right]\right)
$$

using the volume operator $\hat{V}$ of Bianchi models mentioned above and $h_{I}$, $1 \leq I \leq 3$ are the three point holonomies interpreted as multiplication 
operators in a connection interpretation. We also defined

$$
h_{[I, J]}:=\prod_{K=1}^{3}\left(h_{K}\right)^{c_{I J}^{K}}
$$

which is the only place where the constraint operator depends on the Bianchi type (via the structure constants $c_{I J}^{K}$ ). Once we have the Euclidean constraint, the Lorentzian one can be derived completely analogously to the full theory:

$$
\hat{\mathcal{H}}[N]=8 i\left(1+\gamma^{2}\right)\left(\gamma l_{\mathrm{P}}^{2}\right)^{-3} V_{0} N \epsilon^{I J K} \operatorname{tr}\left(\left[h_{I}, \hat{K}\right]\left[h_{J}, \hat{K}\right]\left[h_{K}, \hat{V}\right]\right)-\hat{\mathcal{H}}^{(\mathrm{E})}[N]
$$

using the extrinsic curvature

$$
\hat{K}=i \hbar^{-1}\left[\hat{V}, \hat{\mathcal{H}}^{(\mathrm{E})}[1]\right] .
$$

In order to find a dynamical interpretation of the constraint equation we have to transform from the connection to a dreibein representation and to select an internal time. The transformation to a dreibein representation can be done by expanding an arbitrary homogeneous state $c$ in terms of spin network states labeled by elements $L$ of an index set $\mathcal{I}$ (e.g., three edge spins and the 6-vertex contractor for Bianchi models)

$$
c(A)=\sum_{L \in \mathcal{I}} c_{L} T_{L}(A)
$$

and interpreting the coefficients $c_{L}$ as components of the state in the dreibein representation.

Unfortunately, the volume operator for Bianchi models is quite complicated and cannot be diagonalized explicitly. Therefore, it is awkward to introduce the volume as internal time. However, we can also choose simply one of the edge spins $n$ as internal time (being related to a diagonal component of the homogeneous metric) and still have a discrete time label. Because the Hamiltonian constraint (可) contains holonomies as multiplication operators there are always terms with different 'time' labels $n$ in the action of $\hat{\mathcal{H}}$ on a state. In the dreibein representation we can write

$$
(\hat{\mathcal{H}} c)_{n}=\sum_{i=-\frac{\omega}{2}}^{\frac{\omega}{2}}\left(H_{i} c\right)_{n+\frac{i}{2}}
$$


by collecting on the right hand side all contributions with a fixed spin $n$ (the $H_{i}$ are operators acting on the remaining labels which are suppressed in our notation). The order $\omega$ is given by twice the maximal number of holonomies acting on the selected edge labeled by $n$ which appear in a term of the Hamiltonian constraint operator.

We thus arrive at a Wheeler-DeWitt equation of the form

$$
\sum_{i=-\frac{\omega}{2}}^{\frac{\omega}{2}}\left(H_{i} c\right)_{n+\frac{i}{2}}=0 \quad \text { for all } \quad n \in \frac{1}{2} N_{0}
$$

which is a discrete evolution equation [13] rather than a differential equation as in standard quantum cosmology. Given a set of initial conditions for small $n$ (the number given by the order $\omega$ ) one can compute the evolution as long as the highest operator $H_{n}$ is invertible.

In these models, one can also show that the physical volume spectrum defined using evolving observables is identical to the kinematical one; in particular, it is discrete.

\section{Acknowledgments}

M. B. is grateful to the DFG-Graduierten-Kolleg 'Starke und elektroschwache Wechselwirkung bei hohen Energien' for a PhD fellowship and travel grants.

\section{References}

[1] M. Bojowald and H. A. Kastrup, Class. Quantum Grav. 17 (2000) 3009.

[2] J. Harnad, S. Shnider, and L. Vinet, J. Math. Phys. 21 (1980) 2719.

[3] O. Brodbeck, Helv. Phys. Acta 69 (1996) 321.

[4] A. Ashtekar and J. Lewandowski, Class. Quantum Grav. 14 (1997) A55.

[5] J. D. Bekenstein, Lett. Nuovo Cim. 11 (1974) 467.

[6] J. D. Bekenstein and V. F. Mukhanov, Phys. Lett. B 360 (1995) 7.

[7] M. Bojowald, Class. Quantum Grav. 17 (2000) 1489. 
[8] T. Thiemann, Class. Quantum Grav. 15 (1998) 1487.

[9] M. Bojowald, Class. Quantum Grav. 17 (2000) 1509.

[10] J. J. Halliwell and S. W. Hawking, Phys. Rev. D 31 (1985) 1777.

[11] M. Bojowald, gr-qc/0008052.

[12] T. Thiemann, Class. Quantum Grav. 15 (1998) 839.

[13] M. Bojowald, gr-qc/0008053. 


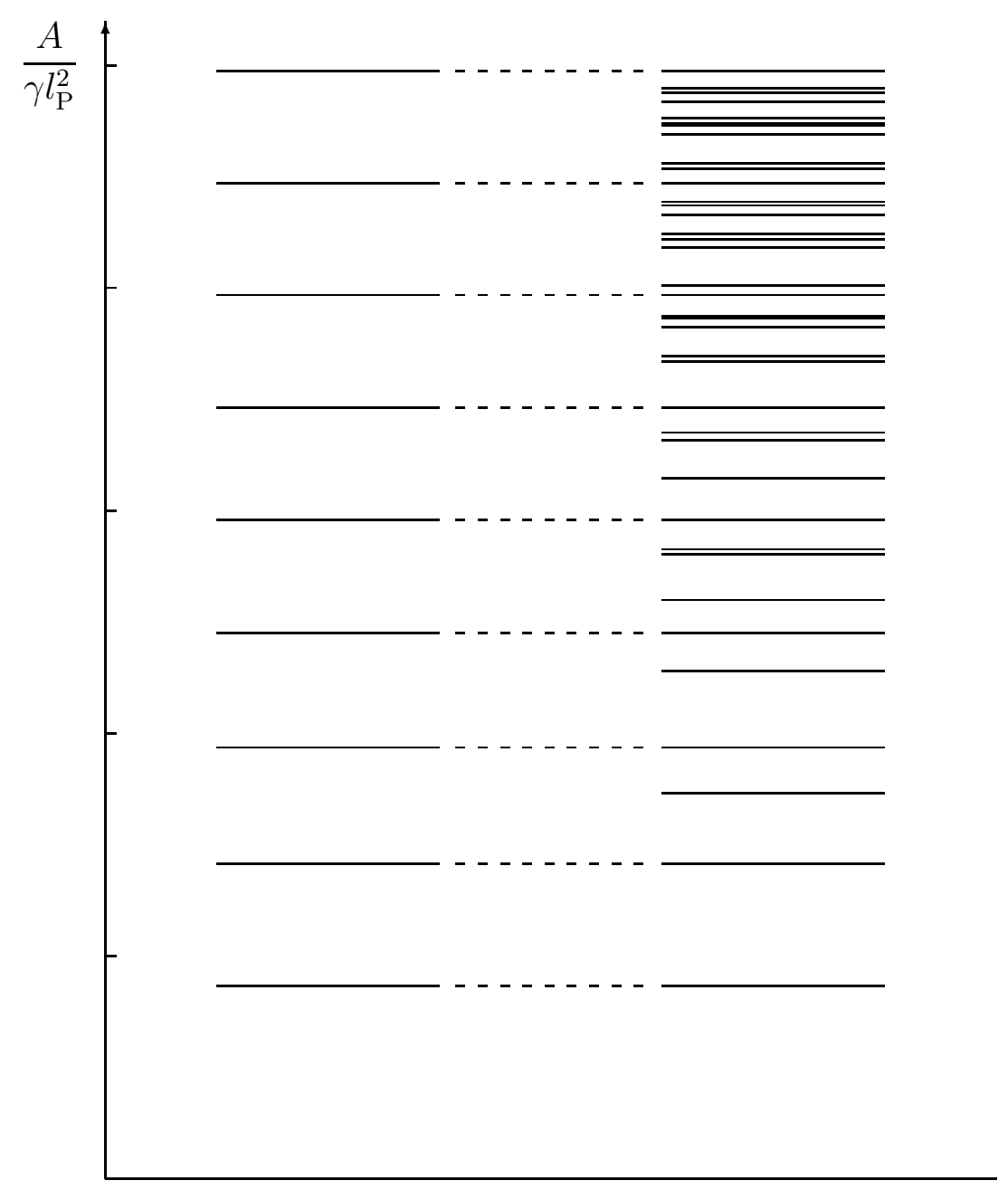

Figure 2: Level Splitting: $\sqrt{j(j+1)}$ (left) vs. $\sum_{p} \sqrt{j_{p}\left(j_{p}+1\right)}$ (right). 